\title{
Sterol uptake and sterol biosynthesis act coordinately to mediate antifungal resistance in Candida glabrata under azole and hypoxic stress
}

\author{
QINGDI QUENTIN LI ${ }^{1}$, HUEI-FUNG TSAI ${ }^{1}$, AJEET MANDAL ${ }^{2}$, BRYAN A. WALKER ${ }^{1}$, \\ JASON A. NOBLE ${ }^{1}$, YUICHI FUKUDA ${ }^{1}$ and JOHN E. BENNETT ${ }^{1}$ \\ ${ }^{1}$ Clinical Mycology Section, Laboratory of Clinical Infectious Diseases, National Institute of Allergy \\ and Infectious Diseases; ${ }^{2}$ Molecular and Cellular Biochemistry Section, National Institute of Dental \\ and Craniofacial Research, National Institutes of Health, Bethesda, MD 20892, USA
}

Received October 11, 2017; Accepted February 23, 2018

DOI: $10.3892 / \mathrm{mmr} .2018 .8716$

\begin{abstract}
Pathogenic fungi, including Candida glabrata, develop strategies to grow and survive both in vitro and in vivo under azole stress. However, the mechanisms by which yeast cells counteract the inhibitory effects of azoles are not completely understood. In the current study, it was demonstrated that the expression of the ergosterol biosynthetic genes $E R G 2, E R G 3, E R G 4, E R G 10$, and ERG11 was significantly upregulated in $C$. glabrata following fluconazole treatment. Inhibiting ergosterol biosynthesis using fluconazole also increased the expression of the sterol influx transporter AUS1 and the sterol metabolism regulators $S U T 1$ and $U P C 2$ in fungal cells. The microarray study quantified 35 genes with elevated mRNA levels, including AUS1, TIR3, UPC2, and $8 E R G$ genes, in a $C$. glabrata mutant strain lacking $E R G l$, indicating that sterol importing activity is increased to compensate for defective sterol biosynthesis in cells. Bioinformatic analyses further revealed that those differentially expressed genes were involved in multiple cellular processes and biological functions, such as sterol biosynthesis, lipid localization, and sterol transport. Finally, to assess whether sterol uptake affects yeast susceptibility to azoles, we generated a $C$. glabrata aus $1 \Delta$ mutant strain. It was shown that loss of Aus1p in C. glabrata sensitized the pathogen to azoles and enhanced the efficacy of drug exposure under low oxygen tension. In contrast, the presence of exogenous cholesterol or ergosterol in medium rendered the $C$.glabrata AUS1 wild-type strain highly resistant
\end{abstract}

Correspondence to: Dr Qingdi Quentin Li or Dr John E. Bennett, Clinical Mycology Section, Laboratory of Clinical Infectious Diseases, National Institute of Allergy and Infectious Diseases, National Institutes of Health, 9000 Rockville Pike, Bethesda, MD 20892, USA

E-mail: quentin.li@nih.gov

E-mail: jbennett@niaid.nih.gov

Key words: Candida glabrata, azole resistance, fluconazole, voriconazole, ergosterol synthesis, sterol uptake to fluconazole and voriconazole, suggesting that the sterol importing mechanism is augmented when ergosterol biosynthesis is suppressed in the cell, thus allowing C. glabrata to survive under azole pressure. On the basis of these results, it was concluded that sterol uptake and sterol biosynthesis may act coordinately and collaboratively to sustain growth and to mediate antifungal resistance in $C$. glabrata through dynamic gene expression in response to azole stress and environmental challenges.

\section{Introduction}

Candida species are ubiquitous fungi and the most common fungal pathogens affecting humans. Candida, in particular, affects high-risk patients who are either immunocompromised or critically ill. More than 100 species of Candida exist, but only a few are recognized as causing disease in humans. Candida glabrata and Candida albicans account for 70 to $80 \%$ of yeasts isolated from patients with invasive candidiasis. In recent decades, $C$. glabrata has become important because of its increasing world-wide incidence and because of its developing resistance to antifungals, including azoles, in the clinic.

Azole antifungal agents such as fluconazole and voriconazole are approved therapy for candidemia and invasive candidiasis caused by $C$. glabrata (1). Mechanisms of azole resistance include i) increased drug export (efflux pumps), ii) lost drug targets (e.g., altered drug target binding site), iii) up-regulated homeostatic stress-response pathways to deal with azole-associated damage, and iv) changed biosynthetic pathways (particularly sterol synthesis) that circumvent or attenuate the effects of azole inhibition (2).

The cellular toxicity of azole antifungals occurs primarily through their ability to affect the fungal cell membrane by inhibiting the biosynthesis of ergosterol, the principal sterol in the fungal cell membrane, leading to the depletion of ergosterol and an accumulation of possibly toxic sterol intermediates in the cytoplasmic membrane. Azole antifungal compounds (imidazoles and triazoles) inhibit cytochrome P-450 dependent sterol $14 \alpha$-demethylase (Erg11p), an enzyme that catalyzes the oxidative removal of the $14 \alpha$-methyl group of lanosterol in 
the ergosterol biosynthetic pathway (2). Fungal cells exposed to azoles must either synthesize more endogenous ergosterol or import exogenous sterol if they are to survive antimycotic treatment.

Sterol uptake appears to confer resistance to antifungal drugs since mutant strains of Candida species lacking AUSI and TIR3, the sterol influx transporters (3), or UPC2, the transcription factor that controls AUS1 and TIR3 expression (3-5), exhibit reduced uptake of cholesterol and are hypersensitive to azoles $(6,7)$, whereas enhanced UPC2 or AUS1 expression and cholesterol uptake have been implicated in the azole-resistant phenotype $(6,7)$. Furthermore, several studies have even suggested that pathogenic fungi can scavenge free sterols for the cell membrane, including cholesterol, resulting in resistance to polyene and azole antifungals (8). Increased uptake of exogenous cholesterol may be associated with drug resistance in clinical isolates of bile-dependent $C$. glabrata cells as well as in sterol auxotrophic $C$. glabrata strains (9). These observations indicate that the ability to scavenge exogenous sterols, when ergosterol biosynthesis is defective or is blocked by antimycotic agents, may play an important role in increased azole resistance in pathogenic fungal species.

Besides sterol uptake, alteration in sterol biosynthesis seems to also affect fungal susceptibility to antifungals. ERG11 is one of the critical genes in the ergosterol biosynthetic pathway, and its encoding protein product Erg11p (or CYP51p depending on nomenclature) is the major target enzyme of azole antifungals. Mutations in ERG11 are the most common mechanism of azole resistance in C. albicans (10). Over-expression of ERG11 has been attributed to decreases in azole activity in $C$. albicans. Alterations in other enzymes of the ergosterol biosynthetic pathway, particularly ERG3 (C-5 sterol desaturase), ERG5 (C-22 sterol desaturase), ERG6 (C-24 sterol methyl-transferase), and ERG25 (C-4 sterol methyloxidase), which are up-regulated with inhibition of Erg11p, have also been documented to reduce azole susceptibility of C. albicans. In C. glabrata, depletion of the ergosterol content in a $C g E R G 1$ mutant increases the levels of susceptibility to azoles, while complementation of the $C g E R G 1$ mutation restores drug sensitivity to wild-type levels (8). In both C. albicans and C. glabrata, regulation of sterol synthesis is vital to azole susceptibility.

In this study, we assessed the role of two protective mechanisms in conferring antifungal susceptibility in $C$. glabrata We showed that expression of the genes involved in both sterol uptake and sterol synthesis is up-regulated in $C$. glabrata when ergosterol is depleted by azole treatment and when ergosterol biosynthesis is defective. We further corroborated that pathogenic fungi can accumulate exogenous sterols from the environment as a protective strategy to survive under azole and hypoxic stress. These findings suggest that sterol uptake and sterol synthesis may act coordinately and collaboratively to sustain growth and to mediate antifungal tolerance in C. glabrata through dynamic gene expression in response to azole pressure and environmental changes.

\section{Materials and methods}

Cell culture and drug treatment. All C. glabrata strains (Table I) used in the present study were cultured on YPD agar containing $1 \%$ Bacto yeast extract (Difco Laboratories, Detroit, MI, USA), 2\% Bacto peptone (Difco Laboratories), and 2\% glucose (Sigma-Aldrich, St. Louis, MO, USA). NCCLS84, Cg1660, CgTn201S, and CgTn201Su/aus1 were also grown on minimal (MIN) agar containing $0.67 \%$ Yeast Nitrogen Base without amino acids (Difco Laboratories) plus $2 \%$ glucose. The ura3 mutants $\mathrm{Cg} 84 \mathrm{u}, \mathrm{Cg} 1660 \mathrm{u}$, and $\mathrm{CgTn} 201 \mathrm{Su}$ were grown in MIN medium supplemented with $20 \mu \mathrm{g} / \mathrm{ml}$ of uracil (Sigma-Aldrich) or were selected on a MIN plus uracil agar plate containing 0.1\% 5-fluoroorotic acid (FOA) (Lancaster, Pelham, NH, USA). YEPG agar was used for drug sensitivity assay, which contained 1\% Bacto yeast extract (Difco Laboratories), 2\% Bacto peptone (Difco Laboratories), 3\% glycerol (Invitrogen/Life Technologies, Carlsbad, CA, USA), $1 \%$ ethanol (Warner-Graham, Inc., Cockeysville, MD), and 2\% agar (Difco Laboratories).

Fluconazole (Euroasian Chemicals Private Ltd., Mumbai, India) was added to cultures of each strain at a final concentration of $200 \mu \mathrm{g} / \mathrm{ml}$, followed by continued incubation with shaking for $2 \mathrm{~h}$. Cell cultures without fluconazole treatment served as controls. After incubation, the cells were harvested, and the cell pellets were stored at $-80^{\circ} \mathrm{C}$ until the subsequent isolation of RNA.

The BBL GasPak Plus gas generator envelope (Becton Dickinson Microbiology Systems, Sparks, MD, USA) or the BBL GasPak Pouch Anaerobic Systems (Becton Dickinson Microbiology Systems, Cockeysville, MD, USA) was used for cultures grown under conditions of low oxygen tension (hypoxia). To mimic the host environment of animals and humans, $C$. glabrata cells were also grown in the presence of $5 \% \mathrm{CO}_{2}$ under low oxygen conditions using an InvivO2 400 workstation at $37^{\circ} \mathrm{C}$ with shaking at $200 \mathrm{rpm}$ (Ruskinn Technology Ltd., Bridgend, UK). For analysis of sterol uptake and exogenous sterol utilization, $2 \mathrm{mg}$ of ergosterol or cholesterol was dissolved in a mixture of $50 \%$ ethanol and $50 \%$ Tween-80 (Sigma-Aldrich) to give a $2 \mathrm{mg} / \mathrm{ml}$ stock solution, which was used to supplement the media with a final sterol concentration of $20 \mu \mathrm{g} / \mathrm{ml}$. For comparison, the same final concentration of ethanol-Tween-80 without sterol was used.

Drug sensitivity assay. The susceptibility of the $C$. glabrata strains Cg1660, CgTn201S, and CgTn201Su/aus1 to fluconazole and voriconazole was determined on MIN and YEPG agar media using an E-test (AB Biodisk, Solna, Sweden) according to the manufacturer's instructions. In brief, logarithmic-phase cells were harvested and adjusted to the desired concentrations by counting the number of cells with a hemocytometer. From each cell suspension, $200 \mu \mathrm{l}\left(1 \times 10^{6}\right.$ cells $\left./ \mathrm{ml}\right)$ was plated in duplicate on MIN and YEPG agar. All plates were incubated under conditions of low oxygen tension at $37^{\circ} \mathrm{C}$ for 3 days. Growth inhibition zones (minimum inhibitory concentration, MIC) to fluconazole and voriconazole were measured.

RT-qPCR analysis. Total RNA was extracted from C. glabrata $(\mathrm{Cg} 84 \mathrm{u})$ logarithmic-phase cultures grown in YPD broth using TRIzol reagent (Invitrogen; Thermo Fisher Scientific, Inc., Waltham, MA, USA) according to the manufacturer's instructions. RNA was converted to cDNA using the High Capacity cDNA Reverse Transcription kit (Applied Biosystems; Thermo Fisher Scientific, Inc.) as previously described (11). Primers 
and probes were designed in our laboratory using the primer analysis software Primer Express 3.0 (Applied Biosystems; Thermo Fisher Scientific, Inc.). TaqMan probes were synthesized by Applied Biosystems (Thermo Fisher Scientific, Inc.) and primers were synthesized by Invitrogen (Thermo Fisher Scientific, Inc.). The sequences of TaqMan probes and forward and reverse primers, as well as the gene numbers for all genes assessed in this study, are listed in Table II. To study gene expression, the amplification was detected in real time using both SYBR Green chemistry (SYBR-Green PCR Master Mix; Applied Biosystems; Thermo Fisher Scientific, Inc.) and TaqMan chemistry (TaqMan Universal PCR Master Mix; Applied Biosystems; Thermo Fisher Scientific, Inc.) as described previously (11).

Microarray hybridization and data analysis. DNA microarray analysis was used to identify genes with altered expression in the C. glabrata ergl mutant strain CgTn201S and the wild-type strain Cg1660. Total RNA was isolated from the log phase culture of $C$. glabrata grown in YPD by using TRIzol (Invitrogen; Thermo Fisher Scientific, Inc.) and the RNeasy MiniElute Cleanup kit (Qiagen, Valencia, CA, USA). RNA was assessed for quality using the Agilent 2100 Bioanalyzer with the RNA 6000 Nano Reagent Kit. Pin-spotted 70-mer oligonucleotide in-house arrays fabricated at the National Institute of Allergy and Infectious Diseases (NIAID) were used for analysis of the pair strains. In brief, a total of 5,908 70-mer oligonucleotides were purchased from Institut Pasteur (Paris, France) and were used for microarray printing at the NIAID Microarray Research Facility. Expression of each open reading frame (ORF) was measured by hybridization to a specific 70-mer oligonucleotide. Thirty micrograms of total RNA from the ergl mutant strain CgTn201S and the wild-type strain Cg1660 was reverse-transcribed to cDNA to incorporate the fluorescent Cy3-dUTP and Cy5-dUTP (GE Healthcare, Piscataway, NJ, USA), respectively. The labeled cDNA of the paired mutant/wild-type strain was combined and used for microarray hybridization. Six microarrays were performed for analysis of the ergl mutant/wild-type pair, including two with reciprocal labeling. The microarrays were prehybridized at $42^{\circ} \mathrm{C}$ in prehybridization buffer [5 x SSC $(1 \times \mathrm{SSC}$ is $0.15 \mathrm{M} \mathrm{NaCl}$ plus $0.015 \mathrm{M}$ sodium citrate), $1 \%$ bovine serum albumin (BSA), $0.1 \% \mathrm{SDS}$ ] for 30 to $60 \mathrm{~min}$ and then hybridized to the labeled cDNA in $50 \mu 1$ of hybridization buffer [25\% formamide, $5 \times \mathrm{SSC}$, $0.2 \%$ SDS, $20 \mu \mathrm{g} / \mathrm{ml}$ poly $(\mathrm{dA})_{40-60}, 200 \mu \mathrm{g} / \mathrm{ml}$ Cot-1 DNA (Invitrogen; Thermo Fisher Scientific, Inc.), $80 \mu \mathrm{g} / \mathrm{ml}$ yeast tRNA] overnight at $42^{\circ} \mathrm{C}$. The microarrays were washed three times in wash buffer A ( $1 \mathrm{x}$ SSC, $0.05 \%$ SDS $)$ and wash buffer B (0.1 x SSC). The in-house arrays were scanned with a GenePix 4000B scanner (Molecular Devices, Sunnyvale, CA, USA). All microarray data archive and statistical calculations were performed on the 'processed signal' data by using the Web-based mAdb analysis system provided by the Bioinformatics and Molecular Analysis group (BIMAS) at the Center for Information Technology (CIT), National Institutes of Health. The data were filtered with the parameters that included genes present in four or more arrays and each array with $80 \%$ or more genes present. The data set of the paired strains was then analyzed by Student's t-test. The genes with P-values less than 0.001 and with at least 1.5 -fold altered gene expression were then selected. The final data set included all genes with altered expression in the pair.

Cloning of CgAUS1. The full nucleotide sequence of the C. glabrata AUS1 homolog (CgAUS1) was obtained from the Candida Genome Database (www.candidagenome.org). A 1,087 bp partial ORF of the CgAUS1 gene was obtained by PCR with PfuUltra High-Fidelity DNA polymerase (Stratagene, La Jolla, California, USA), primer set CgAUS1S (5'-TTGAAGTTGCCTCTTGACTC-3') and CgAUS1AS (5'-AAGGCAACAAACACAGCGGCAG-3'), and the total genomic DNA of $\mathrm{Cg} 1660$ was used as the template. The PCR parameters were $2 \mathrm{~min}$ at $95^{\circ} \mathrm{C}$, followed by 30 cycles of $95^{\circ} \mathrm{C}$ for $30 \mathrm{sec}, 53^{\circ} \mathrm{C}$ or $55^{\circ} \mathrm{C}$ for $30 \mathrm{sec}$, and $72^{\circ} \mathrm{C}$ for $1 \mathrm{~min} 30 \mathrm{sec}$, and then extended at $72^{\circ} \mathrm{C}$ for $10 \mathrm{~min}$. The 1,087 bp PCR product, which was used as a probe for Southern blotting, was then cloned into pCR-Blunt II-TOPO (Invitrogen; Thermo Fisher Scientific, Inc.) to produce plasmid pCgAUS1.

Construction of the Candida glabrata Cgergl and Cgaus1 $\Delta$ double mutant. The Cgerg1 mutant was generated in our laboratory as described previously (8). Gene deletion of Cgaus1 was performed as described by Vermitsky et al (12). Briefly, the primer pair CgAUS1D1S (TTGAAATTCTCGGAAAGA AACATCAAATCAAAAAATTTTAACCTTCTAAAACTT GTTCTTTTTTTGGGAAATATAAGATGTCGAAAGCTA CATATAAGGA) and CgAUS1D2AS (AGTAGATTAAAG AAAAGTGTTAAATTTAAGAATAAAATGGAATTGTTA TTTCATTAAAAGCTTGTAGGAGTCACTCTTATTAGT TTTGCTGGCCGCATCT) was used to generate the CgAUS1 deletion cassette using YEP24 as the template to amplify Saccharomyces cerevisiae URA3, which served as the selection marker. An additional round of PCR using the primer pair CgAUS1D3S (CCCCAACTATCAATTTTCTTTAAATCA AGGAAAATCTATTACATTCGCTATTAATCTCTACTA TCTTTATCTTAGTTTTTTGAAATTCTCGGAAAGAAAC) and CgAUS1D4AS (ATTATATTTTAAATTTTGTTGTAT AGCTTTTTTGCTGTAAAGGTGAAAAAACCGGGAATT TTGAGCATTAGTATTAGTAGATTAAAGAAAAGTGTT AAAT) extended the 5' and 3' ends with about a $160 \mathrm{bp}$ homologous region on each end of the CgAUS1 deletion cassettes to increase the efficiency of homologous recombination. S. cerevisiae URA3 was transformed into CgTn201Su (Cgura3 Cgerg1::Tn5<Cm ura3>) to construct the Cgerg1/aus1 double mutant CgTn201Su/aus1 via a successful double crossover homologous recombination, which resulted in replacement of the CgAUS1 ORF with S. cerevisiae URA3 (Fig. 1A). The $C g E R G 1$ locus in the clinical isolate $\mathrm{Cg} 1660$ and its transposon mutant CgTn201S (Cgerg1) are shown in Fig. 1B. Transformants were selected on MIN medium, and the deletion of CgAUS1 was confirmed by Southern blot analysis of the EcoRI-digested genomic DNA (Fig. 1C). The purified 1.2-kb ScURA3 probe detected a single signal in the Cgergl mutant CgTn201S; two signals were detected in the two putative Cgerg1/aus1 mutants CgTn201Su/aus1-1 and CgTn201Su/aus1-2, while there was no signal in the parental strain $\mathrm{Cg} 1660$ (Fig. 1C, right panel). The 1087-bp CgAUS1 probe detected a single 6.3-kb signal in Cg1660 and CgTn201S, but not in the two putative Cgerg1/aus1 
Table I. Candida glabrata strains used in this study.

\begin{tabular}{|c|c|c|c|}
\hline Strain & Parental strain & Genotype or description & Reference or source \\
\hline NCCLS84 & & Wild-type (ATCC90030) ${ }^{\mathrm{a}}$ & $\mathrm{ATCC}^{\mathrm{a}}$ \\
\hline $\mathrm{Cg} 84 \mathrm{u}$ & NCCLS84 & Cgura3 & $(8)$ \\
\hline Cg1660 & & Clinical isolate & $\mathrm{FHCRC}^{\mathrm{b}}$ \\
\hline $\mathrm{Cg} 1660 \mathrm{u}$ & Cg1660 & Cgura3 & $(8)$ \\
\hline CgTn201S & $\mathrm{Cg} 1660 u$ & Cgura3 Cgerg1::Tn5<Cm URA3> & $(8)$ \\
\hline $\mathrm{CgTn} 201 \mathrm{Su}$ & CgTn201S & Cgura3 Cgerg1::Tn5<Cm ura3> & Present study \\
\hline CgTn201Su/aus1 & $\mathrm{Cg} \operatorname{Tn} 201 \mathrm{Su}$ & $\begin{array}{l}\text { Cgura3 Cgerg1::Tn5<Cm ura3> } \\
\text { Cgaus1 } \Delta:: \mathrm{URA} 3\end{array}$ & Present study \\
\hline
\end{tabular}

${ }^{a}$ American Type Culture Collection (ATCC), Manassas, VA, USA. bHCRC, Fred Hutchinson Cancer Research Center, Seattle, WA, USA.

Table II. Primers and TaqMan probes for RT-qPCR analysis of gene expression used in this study.

\begin{tabular}{|c|c|c|}
\hline Gene & Primer and probe sequence $\left(5^{\prime} \rightarrow 3^{\prime}\right)$ & Gene number ${ }^{\mathrm{a}}$ \\
\hline \multicolumn{3}{|l|}{$A C T 1$} \\
\hline $\mathrm{F}$ & TTGGACTCTGGTGACGGTGTTA & \multirow[t]{3}{*}{ CAGL0K12694g } \\
\hline $\mathrm{R}$ & AAAATAGCGTGTGGCAAAGAGAA & \\
\hline $\mathrm{P}$ & CCACGTTGTTCCAATTTACGCCGG & \\
\hline \multicolumn{3}{|c|}{ RDN5.8 } \\
\hline $\mathrm{F}$ & CTTGGTTCTCGCATCGATGA & \multirow[t]{3}{*}{ CAGL0L13387r } \\
\hline $\mathrm{R}$ & GGCGCAATGTGCGTTCA & \\
\hline $\mathrm{P}$ & ACGCAGCGAAATGCGATACGTAATGTG & \\
\hline \multicolumn{3}{|l|}{$E R G 2$} \\
\hline $\mathrm{F}$ & TCCCAGGTATGACCCATCATC & \multirow[t]{3}{*}{ CAGL0L10714g } \\
\hline $\mathrm{R}$ & TGCGAAGGAGTTTTGATCCAT & \\
\hline $\mathrm{P}$ & ACAAAAGGGCTACGCAAAGCAATACGC & \\
\hline \multicolumn{3}{|l|}{ ERG3 } \\
\hline $\mathrm{F}$ & TGCACTGGCCTCGTGTCTAC & \multirow[t]{3}{*}{ CAGL0F01793g } \\
\hline $\mathrm{R}$ & TAACCGTCGACTGGGTGGAA & \\
\hline $\mathrm{P}$ & TGGTTGGTCTGCACTCCATTCGCC & \\
\hline \multicolumn{3}{|l|}{ ERG4 } \\
\hline $\mathrm{F}$ & CCCTCAATTAGGTGTCGTCATGT & \multirow[t]{3}{*}{ CAGL0A00429g } \\
\hline $\mathrm{R}$ & GGCACGATTAATTCTTCACCCTTA & \\
\hline $\mathrm{P}$ & CCACTGGCTGTACGCTAACGCTTGTG & \\
\hline \multicolumn{3}{|c|}{ ERG10 } \\
\hline $\mathrm{F}$ & GCCAGAACCCCAATTGGTT & \multirow[t]{3}{*}{ CAGL0L12364g } \\
\hline $\mathrm{R}$ & TGCAATGACACCTAGGTCAACAG & \\
\hline $\mathrm{P}$ & TTCCAAGGTGCGTTGGCCTCCA & \\
\hline \multicolumn{3}{|c|}{ ERG11 } \\
\hline $\mathrm{F}$ & TGTCTTGATGGGTGGTCAACA & \multirow[t]{3}{*}{ CAGL0E04334g } \\
\hline $\mathrm{R}$ & CTGGTCTTTCAGCCAAATGCA & \\
\hline $\mathrm{P}$ & CTTCCGCTGCTACCTCCGCTTGG & \\
\hline \multicolumn{3}{|c|}{ PDR16 } \\
\hline $\mathrm{F}$ & CCTGGAGACGTGAATTTGGAAT & \multirow[t]{3}{*}{ CAGL0J07436g } \\
\hline $\mathrm{R}$ & ACAGCAACCAAATCCGATGTAA & \\
\hline $\mathrm{P}$ & CTCGTCACCATTTTCTTCACCCAAATGG & \\
\hline \multicolumn{3}{|c|}{ PDR16 } \\
\hline $\mathrm{F}$ & TTGGCCTGGAGACGTGAATT & \multirow[t]{3}{*}{ CAGL0J07436g } \\
\hline $\mathrm{R}$ & GTTTACCACTTTCATTCTCTACAGCAA & \\
\hline $\mathrm{P}$ & TTGGGTGAAGAAAATGGTGACGAGGTTACA & \\
\hline
\end{tabular}


Table II. Continued.

\begin{tabular}{|c|c|c|}
\hline Gene & Primer and probe sequence $\left(5^{\prime} \rightarrow 3^{\prime}\right)$ & Gene number ${ }^{\mathrm{a}}$ \\
\hline \multicolumn{3}{|l|}{ AUS1 } \\
\hline $\mathrm{F}$ & CCAAGCCACTGCAGGTGAA & \multirow[t]{3}{*}{ CAGL0F01419g } \\
\hline $\mathrm{R}$ & GGCGTGAAACAGGGACTTGA & \\
\hline $\mathrm{P}$ & CGGTGCCCCAACGTCGGGTATC & \\
\hline \multicolumn{3}{|l|}{ SUT1 } \\
\hline $\mathrm{F}$ & GTTGATGGCATTACATGGCAAT & \multirow[t]{3}{*}{ CAGL0I04246g } \\
\hline $\mathrm{R}$ & AGTAAAGGAGTTGGATGATGAGTGAA & \\
\hline $\mathrm{P}$ & ACCAATTCCTATCGCCTCCAATGCCA & \\
\hline \multicolumn{3}{|l|}{ SUT2 } \\
\hline $\mathrm{F}$ & AGGGCCTTCAAGGTATCGAAGT & \multirow[t]{3}{*}{ CAGLOL09383g } \\
\hline $\mathrm{R}$ & TCGGTTTTTGGATCACACCAA & \\
\hline $\mathrm{P}$ & TTGCСТCTCCAAAACAGAAACTACССТССС & \\
\hline \multicolumn{3}{|c|}{$E C M 22$} \\
\hline $\mathrm{F}$ & CAATTACAAGAGCATGCAAACATTG & \multirow[t]{3}{*}{ CAGL0C01199g } \\
\hline $\mathrm{R}$ & GGAGTTAGCCTGACCATGAGTATTATT & \\
\hline $\mathrm{P}$ & TGCATCAGAAACAGCATATCCAACGACTGT & \\
\hline \multicolumn{3}{|c|}{$U P C 2 A$} \\
\hline $\mathrm{F}$ & AAAATAGTACAGGAGCAACGGAGACT & \multirow[t]{3}{*}{ CAGL0C01199g } \\
\hline $\mathrm{R}$ & TGGTTGCACCTGGAGATGAA & \\
\hline $\mathrm{P}$ & CTGTCGCCTTCTCTGAATCTGCTTACACCC & \\
\hline \multicolumn{3}{|c|}{$U P C 2 B$} \\
\hline $\mathrm{F}$ & GGTCGCAAGTGCATTGTTGT & \multirow[t]{3}{*}{ CAGL0F07865g } \\
\hline $\mathrm{R}$ & TCAGTCGCATTTGATGTATCTTTAGG & \\
\hline $\mathrm{P}$ & CGTGGAATAATCACGATCCTCACATGCA & \\
\hline
\end{tabular}

${ }^{a}$ Candida Genome Database (www.candidagenome.org). F, forward primer; R, reverse primer; P, TaqMan probe, labeled as 5'-FAM, 3'-TAMRA; RT-qPCR, reverse transcription-quantitative polyermase chain reaction.

mutants CgTn201Su/aus1-1 and CgTn201Su/aus1-2 (Fig. 1C, left panel).

Southern blot analysis. Candida glabrata genomic DNA was isolated from cultures grown in YPD overnight by using the MasterPure Yeast Purification Kit (Epicentre, Madison, WI, USA). Purified DNA fragments were recovered using the Strataprep Gel DNA Extraction kit (Stratagene; Agilent Technologies, Inc., Santa Clara, CA, USA). Hybond-N nylon membranes (Amersham, Arlington Heights, IL, USA) were used for Southern hybridization analyses. DNA probes were labeled with $\left[\alpha-{ }^{32} \mathrm{P}\right] \mathrm{dCTP}$ or $\left[\alpha-{ }^{32} \mathrm{P}\right] \mathrm{dATP}$ (MP Biomedical, Solon, OH, USA) by using the Prime-It II kit (Stratagene; Agilent Technologies, Inc.). DNA cloning and hybridization analyses were done according to the standard protocol.

Other techniques and reagents. DNA sequencing was done using the DNA sequencing kit with a dRhodamine dye terminator (Applied Biosystems; Thermo Fisher Scientific, Inc.) and an ABI automatic DNA sequencing system (Perkin-Elmer, Foster City, CA, USA). For sequencing of PCR products, PfuUltra DNA polymerase (Stratagene; Agilent Technologies, Inc.) was used for PCR amplification to minimize the rate of
PCR-introduced mutations. The PCR products were cleaned with the Strataprep PCR Purification kit (Stratagene; Agilent Technologies, Inc.) and were used as templates for DNA sequencing.

Bioinformatic and statistical analysis. The Gene Ontology (GO) enrichment analysis and the network visualization of the biological functions related to the differentially expressed genes were performed using Cytoscape software with associated plug-ins (13). To create a functional network by selecting the ClueGO: Function, GO: Biological process, all the network evidence and only the terms with various levels of significance $(\mathrm{P}<0.1-<0.0005)$ were taken into consideration using the plug-in ClueGO (14), which was followed by enrichment of the functional network with the plug-in CluePedia (15).

Results of RT-qPCR for each gene are presented as the fold-change over the expression in the sample not treated with fluconazole, which was set as 1 . The difference in triplicate $\mathrm{C}_{\mathrm{q}}$ values $\left(\Delta \mathrm{C}_{\mathrm{q}}\right)$ was used to calculate the difference between untreated and azole-treated samples. Student's t-test was used to analyze the statistical significance of differences between untreated and fluconazole-treated C. glabrata cells, and P-values were adjusted using Holm's 

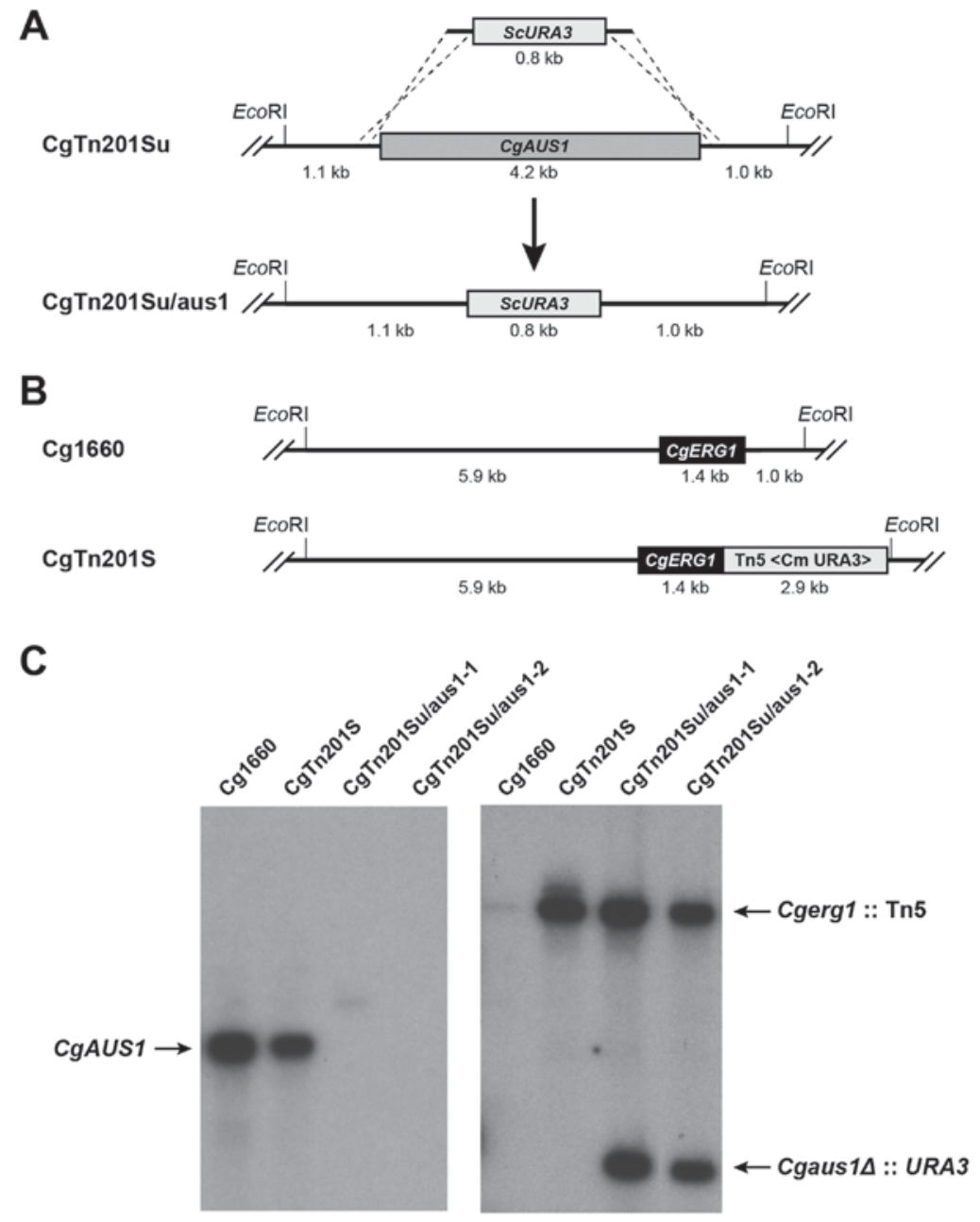

Figure 1. Southern hybridization analysis confirms the deletion and targeted gene replacement of CgAUS1 in Candida glabrata. (A) Deletion strategy of CgAUS1 in the strain CgTn201Su (Cgerg1/ura3). The CgAUS1 ORF was replaced with ScURA3 in the mutant CgTn201Su/aus1 (Cgerg1 Cgaus1 A). (B) The CgERG1 locus in the clinical isolate Cg1660 and its transposon mutant CgTn201S (Cgerg1). (C) Southern blot analysis. The genomic DNAs of Cg1660, CgTn201S, and the mutant CgTn201Su/aus1 were digested with EcoRI, electrophoresed, and blotted onto a nylon membrane. The membrane was hybridized with the 1087-bp CgAUS1 probe (left panel) or 1.2-kb ScURA3 probe (right panel). Lanes (from the left): 1. Cg1660, clinical isolate; 2. CgTn201S, Cgerg1 mutant; 3 . and 4. CgTn201Su/aus1, Cgerg1 Cgaus1 $\Delta$ double mutant. The detected signals for CgAUS1 (left panel) and ScURA3 (right panel) are indicated by arrows.

adjustment for multiple testing. Values of $\mathrm{P}<0.05$ were taken to indicate significance.

\section{Results}

Azole stress up-regulates the genes involved in sterol uptake and biosynthesis. Ergosterol biosynthetic genes encode the enzymes in the sterol synthetic pathway leading to ergosterol biosynthesis in yeast. It is of interest to understand whether the inhibition of ergosterol biosynthesis by azoles alters endogenous sterol synthesis in C. glabrata through changes in the expression of ergosterol biosynthetic genes. We found that mRNA levels of $C g E R G 2, C g E R G 3, C g E R G 4, C g E R G 10$, and $C g E R G 11$ were strikingly increased in C. glabrata following fluconazole treatment. As seen in Table III, the extent of inducible up-regulation of $E R G$ gene expression by fluconazole in these cells ranged from more than 5-fold in $C g E R G 3$ to 19-fold in CgERG10 compared with the untreated controls. Interestingly, we also showed a 3.7-fold increase in mRNA expression of $C g P D R 16$ in fluconazole-treated $C$. glabrata cells (Table III); PDR16p is an ATP-binding cassette (ABC) transporter involved in the sterol biosynthetic process and phospholipid transport in S. cerevisiae (16).

AUS1 and UPC2 (or its paralog ECM22) have been demonstrated to be responsible for sterol uptake in $S$. cerevisiae (17). We next addressed whether inhibition of sterol synthesis by azoles alters the expression of these genes in C. glabrata. Sequencing data have shown that $C$. glabrata is more closely related to Saccharomyces cerevisiae than to C. albicans, and some genes are functionally exchangeable between $C$. glabrata and $S$. cerevisiae. Therefore, to identify AUS1 and ECM22/UPC2 orthologs in C. glabrata, we selected the $S$. cerevisiae AUS1, ECM22, and UPC2 genes as queries to perform homology searches. A BLAST search against the $C$. glabrata open reading frame (ORF) nucleotide and amino acid sequences (www.candidagenome.org) predicted CAGL0F01419g (CgAUS1) to be the ortholog of AUS1. We also performed a BLAST search against $C$. glabrata ORF nucleotide sequence alone, which predicted CAGLOC01199g and CAGL0F07865g to be possible orthologs of ECM22 or UPC2. The amino acid sequences encoded by these two genes are particularly highly homologous to Upc2p or Ecm22p. We refer 
to CAGL0C01199g and CAGL0F07865g as UPC2A (ECM22) and $U P C 2 B$ (ECM22), respectively. This is in concordance with the work of Nagi et al (4) and Whaley et al (18). Our results demonstrate a transcriptional up-regulation of over two-fold and over four-fold in $C g A U S 1$ and $C g U P C 2 A$ expression, respectively, in our model following fluconazole challenge (Table III). These data clearly show that inhibition of ergosterol synthesis by azoles induce the expression of sterol transporter genes and likely lead to enhanced sterol uptake in C. glabrata.

Besides its role in anaerobic sterol uptake, UPC2 has been shown to be an activator of ergosterol biosynthetic genes in yeast $(4,18)$. As the up-regulation of $C g U P C 2$ expression was induced by fluconazole in $C$. glabrata, this result prompted us to address whether azoles alter the expression of other genes encoding transactivators responsible for sterol synthesis or uptake in fungi. It has been demonstrated that SUTI is a transcription factor involved in sterol uptake and synthesis in aerobically growing $S$. cerevisiae cells (19). Therefore, to identify $S U T 1$ ortholog in C. glabrata, we selected the S. cerevisiae $S U T 1$ gene as queries to perform homology searches. A BLAST search against the $C$. glabrata $\mathrm{ORF}$ nucleotide sequence (www.candidagenome.org) predicted CAGL0I04246g and CAGL0L09383g to be possible orthologs of SUT1. The amino acid sequences encoded by these two genes are particularly highly homologous to Sut1p. We refer to CAGL0I04246g and CAGLOL09383g as $C g S U T 1$ and $C g S U T 2$, respectively. In the current model, we show that the expression of $C g S U T 1$, but not $C g S U T 2$, was significantly increased by fluconazole in C. glabrata (Table III).

Up-regulated expression of the genes involved in sterol uptake and biosynthesis in Candida glabrata ergl mutant. To further assess whether ergosterol depletion affects the genes involved in sterol uptake and sterol synthesis in C. glabrata, we used DNA microarray to analyze gene expression in a $C$. glabrata ergl mutant with defective ergosterol biosynthesis. Transcriptional profiling of the microarrays revealed 35 genes up-regulated and 4 genes down-regulated in the $C$. glabrata ergl mutant CgTn201S compared to the parental wild-type strain Cg1660. Particularly, C. glabrata ergl mutant had 2.5-fold up-regulation of CgAUS1 mRNA, 5.6-fold up-regulation of CgTIR3 mRNA, and 3.5-fold up-regulation of $C g U P C 2 B$ mRNA over the wild-type cells (Fig. 2). To a lesser extent, we also found that the up-regulation of $C g E R G 2, C g E R G 3, C g E R G 5$, CgERG6, CgERG7, CgERG10, CgERG11, and CgERG29 in the $C$. glabrata ergl mutant cells ranged from 1.5-to 2.8-fold mRNA expression among these genes (Table IV), which is similar to our data from the C. glabrata cells in which ergosterol synthesis was inhibited by fluconazole (Table III). Additionally, we show a 3-fold increase in mRNA expression of $H E S 1 / K E S 1$, which is involved in ergosterol biosynthesis, oxysterol binding, and sterol transport (Table IV). These data highlight the notion that blocking ergosterol synthetic pathway leading to ergosterol depletion causes increased sterol uptake activity and sterol biosynthesis in C. glabrata.

Besides the genes for sterol uptake and synthesis, 23 other genes demonstrated dominant and reproducible expression in C. glabrata ergl mutant with the average level of the six microarrays ranging from 1.5 -fold to 6.3 -fold greater than the wild-type strain (Table IV). These differentially expressed
Table III. Upregulated expression of sterol biosynthetic and sterol transporter genes in Candida glabrata under fluconazole stress.

\begin{tabular}{lc}
\hline Gene & mRNA levels \\
\hline$E R G 2$ & $11.79^{\mathrm{a}}$ \\
ERG3 & $5.04^{\mathrm{a}}$ \\
ERG4 & $11.08^{\mathrm{a}}$ \\
ERG10 & $19.07^{\mathrm{a}}$ \\
ERG11 & $5.87^{\mathrm{a}}$ \\
PDR16 & $3.73^{\mathrm{a}}$ \\
AUS1 & $2.51^{\mathrm{b}}$ \\
SUT1 & $2.60^{\mathrm{b}}$ \\
SUT2 & 0.97 \\
UPC2A & $4.71^{\mathrm{a}}$ \\
UPC2B & $1.60^{\mathrm{b}}$ \\
\hline
\end{tabular}

Values indicate the fold-change in RNA transcription for each gene in $200 \mu \mathrm{g} / \mathrm{ml}$ fluconazole-treated C. glabrata after normalization to RDN5.8 as the reference, as compared with the level in untreated cells, which was set as $1 .{ }^{\mathrm{b}} \mathrm{P}<0.05$ or ${ }^{\mathrm{P}}<0.01$ between fluconazole-treated and untreated $C$. glabrata cells for all values shown in this table.

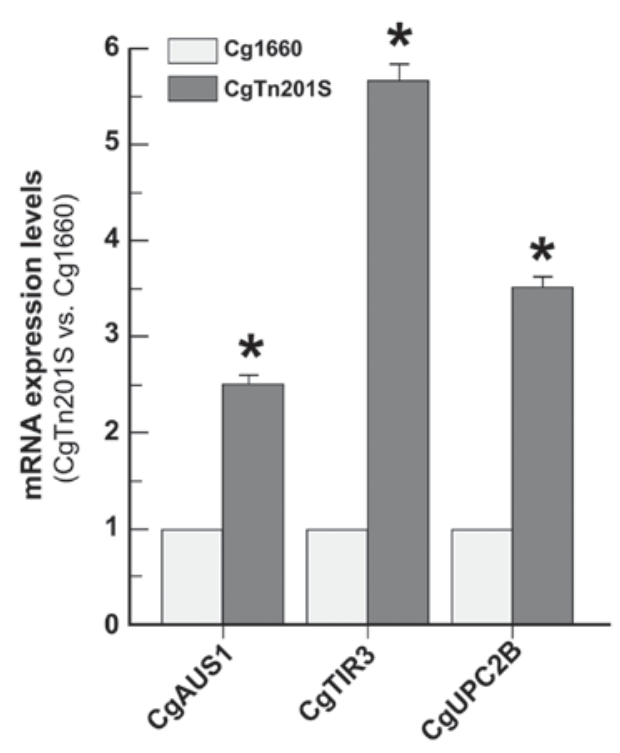

Figure 2. Expression of $C g A U S 1, C g T I R 3$, and $C g U P C 2 B$ is up-regulated in Candida glabrata erg1 mutant. DNA microarray was used to analyze gene expression in the C. glabrata erg1 mutant CgTn201S and its parental wild-type strain Cg1660 as described in Materials and methods. Six microarrays were performed for analysis of the Cgerg1 mutant/wild-type pair, including two with reciprocal labeling. The average mRNA expression levels were based on mean of six replicates. Transcriptional profiling of the microarrays revealed the up-regulation (fold-change) of $\mathrm{CgAUS1}, \mathrm{CgTIR} 3$, and $C g U P C 2 B$ in CgTn201S (Cgerg1) as compared with Cg1660 (wild-type), which was set as $1 .{ }^{*} \mathrm{P}<0.01$ between the $C$. glabrata erg1 mutant $\mathrm{CgTn} 201 \mathrm{~S}$ and its parental wild-type strain Cg1660 using Student's t-test.

genes were associated with multiple different cellular metabolisms and biological processes, such as iron, amino acid, and carbohydrate metabolisms, alcohol metabolism, reactive oxygen species (ROS) metabolism, heme biosynthesis, biotin biosynthesis, mitochondrion organization, mitochondrial electron 
Table IV. Candida glabrata genes up- and down-regulated $\geq 1.5$-fold in response to CgERG1 disruption (Cgerg 1 mutation) in Cg1660 host.

$\begin{array}{lcc}\text { C. glabrata } & \begin{array}{c}\text { S. cerevisiae } \\ \text { homologue }\end{array} & \\ \text { designation } & \text { Description } & \text { Fold expression } \\ \end{array}$

Upregulated genes

CAGLOF01419g

CAGLOC03872g

AUS1

CAGLOF07865g

TIR3/YIL011w

UPC2B

CAGLOL10714g

ERG2

CAGL0F01793g

ERG3

CAGL0M07656g

ERG5

CAGLOH04653g

ERG6

CAGLOJ10824g

ERG7

CAGLOL12364g

\section{ERG10/POT14}

CAGLOE04334g

ERG11

CAGLOK03927g

ERG29

CAGL0J03916g

HES1/KES 1

CAGLOJ00297g

YHR045w

CAGL0A01089g

YPL272c

CAGLOI01408g

CAGLOL03828g

CYC1

CAGL0K10868g

CYB5

CTA1

CAGL0K12100g

HEM13

CAGL0G03905g

ISA1

CAGLOH04851g

PPZ1

CAGLOL07480g

NRG1/NRG2

CAGLOF01485g

TIR4

CAGLOH09614g

TIR1

CAGLOC00110g

FLO1

CAGLOM04125g

YNL320w
ATP-binding cassette transporter

2.5006

involved in sterol uptake

Putative GPI-linked cell wall protein

involved in sterol uptake

Transcription factor transcriptionally

regulates ergosterol biosynthetic genes

and sterol transporter genes

C-8 sterol isomerase participates in ergosterol

biosynthesis

C-5 sterol desaturase participates in

ergosterol biosynthesis

C-22 sterol desaturase participates in

ergosterol biosynthesis

C-24 sterol methyltransferase participates

in ergosterol biosynthesis

Lanosterol synthase participates in

ergosterol biosynthesis

Acetyl-CoA C-acetyltransferase participates

in ergosterol biosynthesis

Lanosterol 14-a-demethylase involved

in ergosterol biosynthesis

Roles in ergosterol biosynthesis,

mitochondrion organization, etc

Roles in ergosterol biosynthesis, oxysterol binding, sterol transport, etc

Possible roles in iron, amino acid,

5.6547

3.4979

2.0136

2.7363

2.3415

2.1176

2.31

2.2113

2.8477

1.5044

2.9779

1.5889

and carbohydrate metabolisms

Alcohol O-acetyltransferase with role

4.0299

in alcohol metabolic process

Cytochrome- $c$ isoform 1 involved in

2.6164

mitochondrial electron transport

Cytochrome b5 involved in

1.5483

oxidation-reduction process

Catalase A involved in cellular

2.7464

response to oxidative stress

Coproporphyrinogen III oxidase

2.3296

involved in heme biosynthesis

Regulation of ROS metabolic process

1.5708

and biotin biosynthetic process

Protein phosphatase $\mathrm{Z}$ involved in

cation homeostasis and cell wall integrity

Transcription factor activity,

1.613

sequence-specific DNA binding activity

Putative GPI-linked cell wall

5.4181

mannoprotein of the Srp1p/Tip1p family

Putative GPI-linked cell wall protein

6.3371

Member of the FLO family of

1.9697

cell wall flocculation proteins

Roles in cell polarity, endoplasmic reticulum, mitochondrion, etc 
Table IV. Continued.

\begin{tabular}{|c|c|c|c|}
\hline $\begin{array}{l}\text { C. glabrata } \\
\text { designation }\end{array}$ & $\begin{array}{l}\text { S. cerevisiae } \\
\text { homologue }\end{array}$ & Description & Fold expression ${ }^{\mathrm{a}}$ \\
\hline CAGL0E00187g & YMR317w & $\begin{array}{l}\text { Putative adhesin-like protein; belongs } \\
\text { to adhesin cluster IV }\end{array}$ & 4.3814 \\
\hline CAGL0G04499g & SET4/YJL105w & Ortholog of S. cerevisiae: SET4 and YJL105w & 1.9778 \\
\hline CAGL0F08965g & MSC7/YHR039c & $\begin{array}{l}\text { Roles in cytosol, endoplasmic reticulum, } \\
\text { nucleus localization, etc }\end{array}$ & 1.6906 \\
\hline CAGL0C00209g & DAN1/YJR151c & $\begin{array}{l}\text { Putative adhesin-like cell wall protein; } \\
\text { predicted GPI-anchor }\end{array}$ & 4.6475 \\
\hline CAGL0G10175g & DAN1/YJR151c & $\begin{array}{l}\text { Adhesin-like protein; predicted } \\
\text { GPI anchor }\end{array}$ & 4.7887 \\
\hline CAGL0K04279g & SCM4/YGR049w & $\begin{array}{l}\text { Ortholog(s) have mitochondrial } \\
\text { outer membrane localization }\end{array}$ & 1.5542 \\
\hline \multicolumn{4}{|l|}{$\begin{array}{l}\text { Downregulated } \\
\text { genes }\end{array}$} \\
\hline CAGL0H03971g & YCP4/PST2 & $\begin{array}{l}\text { Roles in cellular response to oxidative stress, } \\
\text { mitochondrion, etc }\end{array}$ & 1.5484 \\
\hline CAGL0M05995g & PET10 & $\begin{array}{l}\text { Roles in lipid metabolism, respiratory growth, } \\
\text { and ATP/ADP exchange }\end{array}$ & 1.5975 \\
\hline CAGL0G05566g & FMP45 & $\begin{array}{l}\text { In mitochondria; role in ascospore formation, } \\
\text { cellular response to drug, etc }\end{array}$ & 1.5862 \\
\hline CAGL0L10142g & RSB1/YOR049c & $\begin{array}{l}\text { Sphingolipid transporter; involved in fatty } \\
\text { acid transport }\end{array}$ & 2.1631 \\
\hline
\end{tabular}

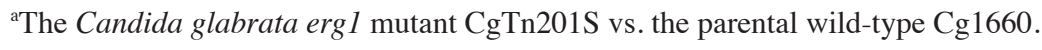

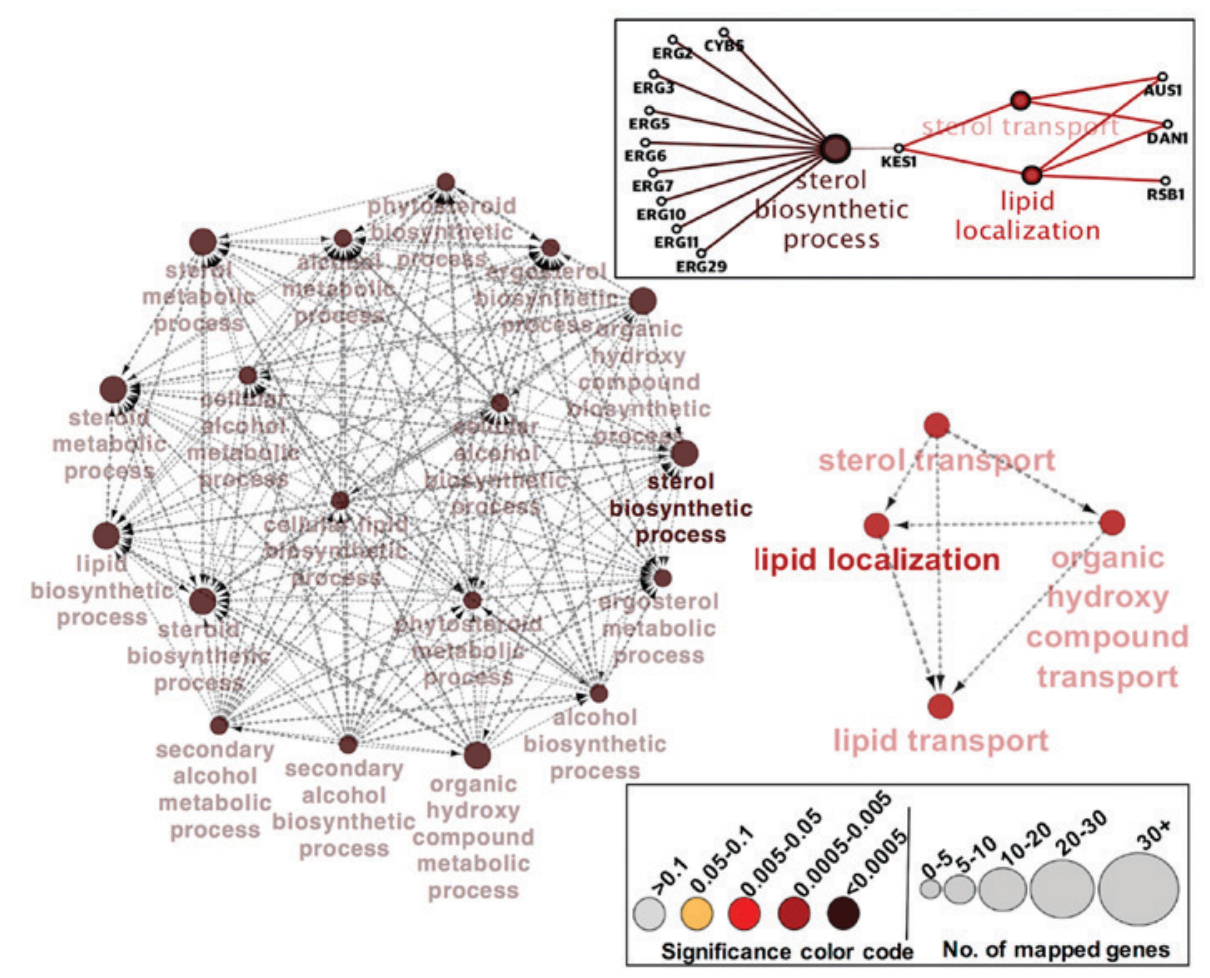

Figure 3. Two major functional networks obtained from the 35 differentially expressed genes in Candida glabrata erg1 mutant using Cytoscape software. Each node (filled circle) represents a biological process and the size and color code indicate, respectively, the number of genes mapped and the significance of the terms (lower right inset). The direction of the network is shown by arrow-head of edges; the edge-thickness is based on kappa-score level calculated automatically by ClueGO. The molecular interaction network between the 'sterol biosynthetic process' and 'lipid localization' in combination with 'sterol transport' is shown in the upper right inset. 
Table V. Functional enrichment with associated genes identified by using Cytoscape and GO database.

\begin{tabular}{lll}
\hline Enriched process & \multicolumn{1}{c}{ Associated gene } & P-value \\
\hline Sterol biosynthetic process & $C Y B 5, E R G 2, E R G 3, E R G 5, E R G 6$, & $<0.0005$ \\
Lipid localization & $E R G 7, E R G 10, E R G 11, E R G 29$ & 0.0005 to 0.005 \\
\hline
\end{tabular}

GO, Gene Ontology.

transport, oxidation-reduction process, cation homeostasis, cell wall integrity, and in-cell polarity, endoplasmic reticulum, mitochondrion, nucleus localization, and so forth (Table IV). Furthermore, mRNA expression of 4 genes was down-regulated 1.5- to 2.2-fold lower than the parental wild-type cells (Table IV). These down-regulated genes were enriched in several cellular and metabolic processes, including lipid metabolism, sphingolipid and fatty acid transport, respiratory growth, ATP/ADP exchange, and cellular response to oxidative stress (Table IV).

Potential coordination between sterol biosynthesis and sterol uptake in Candida glabrata ergl mutant. To further explore the functional themes of the above 35 differentially expressed genes in C. glabrata ergl mutant, we used Cytoscape, a bioinformatics software for visualizing functional and molecular interaction networks. The functional interaction networks in Fig. 3 support our interpretation that the ergosterol biosynthetic process is the major biological function affected in C. glabrata mutant strain lacking ERG1. To compensate for the defective sterol biosynthetic pathway in the cell, and overcome the imbalance in cellular levels of sterol, cells increase the sterol uptake machinery and ergosterol biosynthesis processes in order to survive under azole and environmental stress. As shown in Fig. 3, nine genes (ERG2, ERG3, ERG5, ERG6, ERG7, ERG10, ERG11, $E R G 29$, and $C Y B 5$ ) comprise the largest network cluster and contribute towards the enrichment of the 'sterol biosynthetic process.' While three genes (AUS1, DAN1, and RSB1) in the other smaller functional network cluster are enriched with 'lipid localization' in combination with GO-term 'sterol transport' (Fig. 3). KES1 and HES1 are the genes bridging the two major clusters by establishing a potential coordination between the 'sterol biosynthetic process' and 'lipid localization/sterol transport' (Fig. 3). The enriched processes identified with the Cytoscape software and GO database using the differentially expressed genes in C. glabrata ergl mutant are shown in Table V.

Candida glabrata ausl deletant is defective in sterol uptake and has greater susceptibility to azoles under hypoxic conditions. Lastly, we validated whether $C$. glabrata cells accumulate sterols from the environment through the Aus1p transporter and whether sterol uptake confers resistance to azoles in C. glabrata. To distinguish the uptake of exogenous sterol from that of endogenous ergosterol biosynthesis, we generated a $C$. glabrata erg1/aus $1 \Delta$ double mutant strain and used the $C$. glabrata ergl mutant strain CgTn201S and the wild-type strain Cg1660 as the controls. As seen in
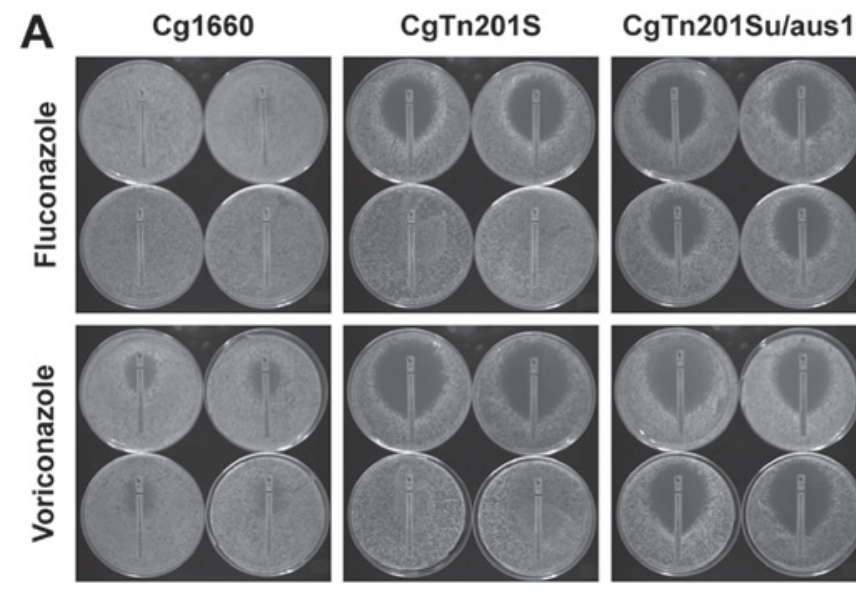

B
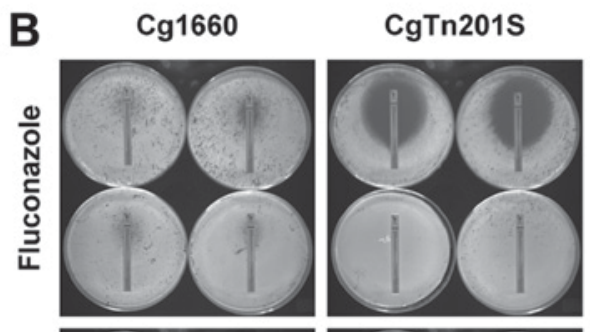

CgTn201Su/aus1
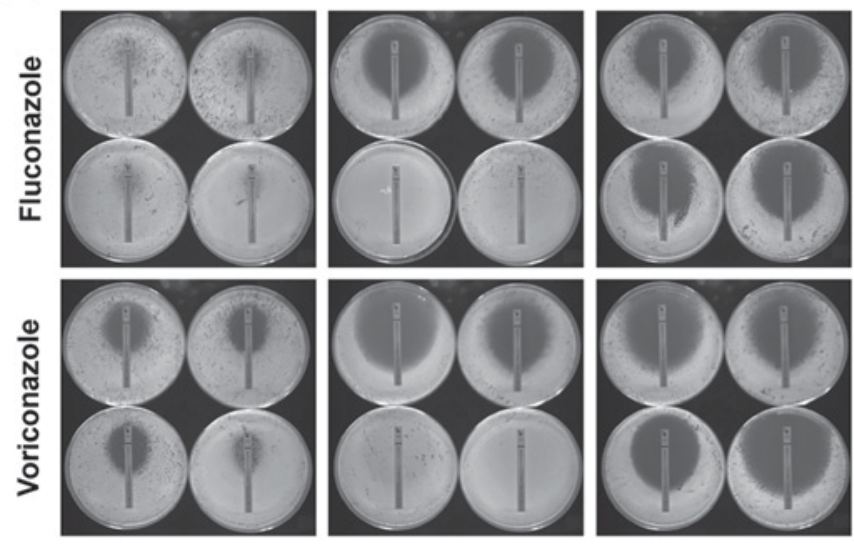

Figure 4. CgAUS1 deletion in Candida glabrata abolishes the effect of sterol supplement on azole susceptibility under hypoxic conditions. Cg1660 (wild-type), CgTn201S (Cgerg1), or CgTn201Su/aus1 (Cgerg1/aus14) were grown on (A) MIN agar medium or (B) YEPG agar medium. Plates were incubated under conditions of low oxygen tension at $37^{\circ} \mathrm{C}$ for 3 days. The fluconazole and voriconazole susceptibilities of Cg1660, CgTn201S, and $\mathrm{CgTn} 201 \mathrm{Su} / \mathrm{aus} 1$ were analyzed by E-test. The intercept of the zone of growth inhibition with the paper strip indicates the minimum inhibitory concentration (MIC). The addition of cholesterol or ergosterol is indicated by the position within each of the four groups as follows: upper left corner, MIN or YEPG; upper right corner, MIN or YEPG with ethanol-Tween 80 solvent alone; lower left corner, MIN or YEPG with cholesterol; and lower right corner, MIN or YEPG with ergosterol. The numerical data are shown in Tables VI and VII for MIN agar cultures and YEPG agar cultures, respectively.

Fig. 4A and B and Tables VI and VII, in the presence of exogenous cholesterol or ergosterol, $C$. glabrata erg1 mutant cells showed much lower susceptibility to fluconazole and voriconazole compared to those cells in the absence of exogenous sterols, suggesting that $C$. glabrata ergl mutant cells are 
Table VI. Inhibitory effect of azoles in Candida glabrata wild-type, erg1 mutant, and erg $1 /$ aus $1 \Delta$ double mutant on MIN agar medium with sterol supplement under hypoxic condition.

E-test on MIN agar medium

\begin{tabular}{|c|c|c|c|c|c|}
\hline Strain $^{a}$ & $\begin{array}{c}\text { Detergent }(0.5 \% \text { EtOH } \\
\text { and } 0.5 \% \text { Tween-80) }\end{array}$ & $\begin{array}{l}\text { Cholesterol } \\
(20 \mu \mathrm{g} / \mathrm{ml})\end{array}$ & $\begin{array}{l}\text { Ergosterol } \\
(20 \mu \mathrm{g} / \mathrm{ml})\end{array}$ & $\begin{array}{l}\text { Fluconazole MIC } \\
\qquad(\mu \mathrm{g} / \mathrm{ml})\end{array}$ & $\begin{array}{l}\text { Voriconazole MIC } \\
\qquad(\mu \mathrm{g} / \mathrm{ml})\end{array}$ \\
\hline $\operatorname{Cg} 1660$ & - & - & - & $>256$ & 4.0 \\
\hline Cg1660 & + & - & - & $>256$ & 4.0 \\
\hline Cg1660 & + & + & - & $>256$ & 12 \\
\hline Cg1660 & + & - & + & $>256$ & 12 \\
\hline CgTn201S & - & - & - & 0.50 & 0.064 \\
\hline CgTn201S & + & - & - & 0.50 & 0.064 \\
\hline CgTn201S & + & + & - & $>256$ & $>32$ \\
\hline CgTn201S & + & - & + & $>256$ & $>32$ \\
\hline CgTn201Su/Cgaus1 & - & - & - & 0.50 & 0.064 \\
\hline CgTn201Su/Cgaus1 & + & - & - & 0.50 & 0.064 \\
\hline CgTn201Su/Cgaus1 & + & + & - & 0.50 & 0.064 \\
\hline CgTn201Su/Cgaus1 & + & - & + & 0.50 & 0.064 \\
\hline
\end{tabular}

${ }^{a}$ Under hypoxic conditions $\left(1 \% \mathrm{O}_{2}\right)$. Cg1660, C. glabrata wild-type; CgTn201S, C. glabrata erg1 mutant; CgTn201S/Cgaus1, C. glabrata erg 1/aus1 $\triangle$ double mutant; EtOH, ethanol; MIC, minimum inhibitory concentration.

Table VII. Inhibitory effect of azoles in Candida glabrata wild-type, erg1 mutant, and erg 1/aus1 $\Delta$ double mutant on YEPG agar medium with sterol supplement under hypoxic condition.

\begin{tabular}{|c|c|c|c|c|c|}
\hline \multirow[b]{2}{*}{ Strain $^{\mathrm{a}}$} & \multicolumn{5}{|c|}{ E-test on YEPG agar medium } \\
\hline & $\begin{array}{c}\text { Detergent }(0.5 \% \text { EtOH } \\
\text { and } 0.5 \% \text { Tween-80) }\end{array}$ & $\begin{array}{l}\text { Cholesterol } \\
(20 \mu \mathrm{g} / \mathrm{ml})\end{array}$ & $\begin{array}{l}\text { Ergosterol } \\
(20 \mu \mathrm{g} / \mathrm{ml})\end{array}$ & $\begin{array}{l}\text { Fluconazole MIC } \\
\qquad(\mu \mathrm{g} / \mathrm{ml})\end{array}$ & $\begin{array}{l}\text { Voriconazole MIC } \\
\qquad(\mu \mathrm{g} / \mathrm{ml})\end{array}$ \\
\hline $\operatorname{Cg} 1660$ & - & - & - & $>256$ & 1.0 \\
\hline Cg1660 & + & - & - & $>256$ & 1.0 \\
\hline Cg1660 & + & + & - & $>256$ & 1.0 \\
\hline $\operatorname{Cg} 1660$ & + & - & + & $>256$ & 6.0 \\
\hline CgTn201S & - & - & - & 1.0 & 0.064 \\
\hline CgTn201S & + & - & - & 1.0 & 0.064 \\
\hline CgTn201S & + & + & - & $>256$ & $>32$ \\
\hline CgTn201S & + & - & + & $>256$ & $>32$ \\
\hline CgTn201Su/Cgaus1 & - & - & - & 1.0 & 0.064 \\
\hline CgTn201Su/Cgaus1 & + & - & - & 1.0 & 0.064 \\
\hline CgTn201Su/Cgaus1 & + & + & - & 1.0 & 0.064 \\
\hline CgTn201Su/Cgaus1 & + & - & + & 1.0 & 0.064 \\
\hline
\end{tabular}

${ }^{\mathrm{a} U n d e r}$ hypoxic conditions $\left(1 \% \mathrm{O}_{2}\right)$. Cg1660, C. glabrata wild-type; CgTn201S, C. glabrata erg1 mutant; CgTn201S/Cgaus1, C. glabrata erg 1/aus1 $\Delta$ double mutant; EtOH, ethanol; MIC, minimum inhibitory concentration.

capable of accumulating sterols from the medium through the wild-type sterol transporter CgAus1p; but not through ergosterol synthesis because the cells were defective in ergosterol synthetic pathway. In contrast, in the presence of exogenous cholesterol or ergosterol, the $C$. glabrata erg1/aus $1 \Delta$ double mutant shows much higher susceptibility to fluconazole and voriconazole than the $C$. glabrata ergl mutant, indicating that $C$. glabrata erg1 1 aus $1 \Delta$ double mutant cells are not able to uptake and synthesize sterols due to defective conditions in both the CgAus1p transporter and ergosterol biosynthesis in the cells. We also tested whether the loss of CgAus1p in C. glabrata sensitizes the pathogen to polyene amphotericin $\mathrm{B}$ and the echinocandins anidulafungin and caspofungin, and our E-test results reveal that $C$. glabrata cells lacking CgAus1p do not show altered susceptibility to non-azole antifungals in the presence or absence of exogenous sterols (data not shown). These observations confirm the findings of Zavrel et al (20) that CgAus1p is responsible for taking up exogenous cholesterol or ergosterol in C. glabrata under hypoxic stress when sterol synthesis is either absent or 
insufficient, and further suggest that sterol uptake plays an important role in the development of azole resistance in yeast under ergosterol starvation conditions, such as under azole drug pressure or when defective in sterol biosynthesis.

\section{Discussion}

Yeast develops strategies to grow and survive in different unfavorable environments. In the present study, we observed that $C$. glabrata acquires two protective mechanisms to allow the pathogen to grow and survive under azole and hypoxic stress: Through increasing endogenous sterol synthesis or through importing exogenous sterols. Our data revealed that the expression of both ergosterol biosynthesis and sterol metabolism regulator genes, as well as sterol influx transporter genes, are significantly increased in C. glabrata under fluconazole stress. Likewise, the sterol influx transporter genes ( $C g A U S 1$ and $C g T I R 3)$ and the ergosterol biosynthetic genes (CgERG2, CgERG3, CgERG5, CgERG6, CgERG7, CgERG10, CgERG11, and CgERG29) are markedly up-regulated in yeast when ergosterol biosynthesis is suppressed either under hypoxia or due to defective sterol synthesis. We also confirmed that CgAUS1 in the cell is responsible for importing exogenous cholesterol and ergosterol, thus allowing $C$. glabrata to survive under low oxygen tension conditions or under azole pressure. The presence of both in vivo suggests an underlying mechanism for azole resistance in clinical practice.

Nakayama and colleagues show that CgAUS1 protects C. glabrata cells against azoles in the presence of serum (21). The composition of serum is complicated, and it includes many different molecules, minerals, and nutrients other than cholesterol. It is possible that, besides importing cholesterol via the sterol transporter CgAUS1, C. glabrata cells may also take up other molecules and nutrients from serum that are necessary to their survival amidst azole treatment. In our experiments, as in those of Zavrel et al (20), we did not use serum but solubilized cholesterol and ergosterol using the detergent Tween 80. This allowed for the incorporation of exogenous sterol into the fungal cell. Our results, together with the data discussed above, reinforce the understanding of how both enhanced endogenous sterol synthesis and increased exogenous sterol uptake by yeast are integral to conferring resistance to azole therapy in fungal infections. Resistance to azole treatment not only limits the usefulness of this class of drugs, it also drives the survival of intrinsically low-susceptibility $C$. glabrata cells that become increasingly resistant following prolonged treatment with azole therapeutic agents.

Combining our data in this study, we propose a hypothetical model in which sterol uptake and sterol biosynthesis act coordinately and collaboratively to mediate azole antifungal resistance in $C$. glabrata under azole and hypoxic stress as shown in Fig. 5. In this model, azole and hypoxic stresses deplete ergosterol directly or indirectly by inhibiting ergosterol biosynthesis. Hypoxia and azoles cause mitochondrial dysfunction by depriving the mitochondria of oxygen and inducing the accumulation of toxic sterol-intermediates, ultimately leading to the reduction of ergosterol biosynthesis. In response, ergosterol depletion triggers up-regulation of the genes involved in sterol transport and ergosterol biosynthesis,

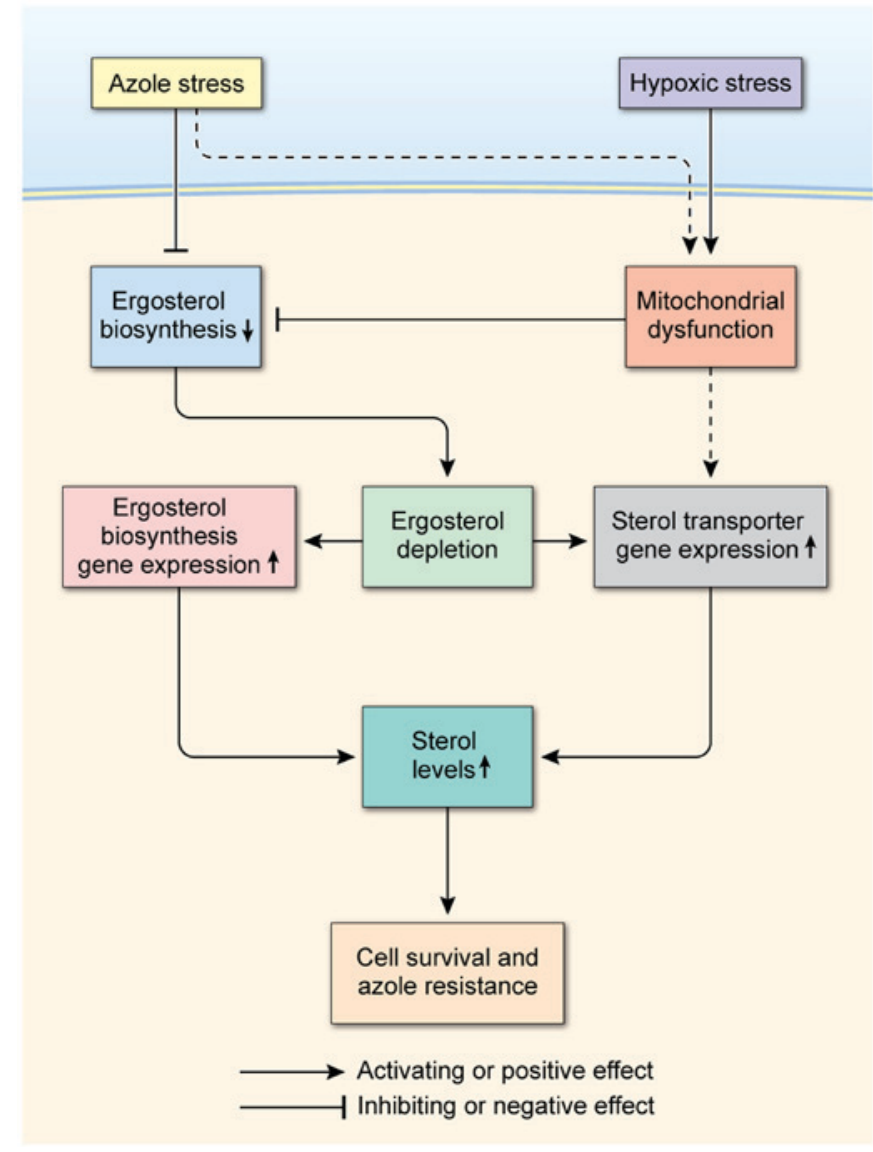

Figure 5. A schematic diagram illustrating a hypothetical model for sterol uptake and sterol biosynthesis acting coordinately and collaboratively in mediating azole antifungal resistance in Candida glabrata under azole and hypoxic stress. See 'Discussion' for further elaboration.

leading to increases in sterol levels. Because both sterol uptake and sterol biosynthesis increase sterol levels through distinct mechanisms, they both may function together to maintain sterol levels and sustain cell growth. Such a cooperative mechanism may serve to integrate the roles of sterol transport and sterol biosynthesis culminating in cell survival, which may underlie the mechanism of azole antifungal resistance in C. glabrata.

Collectively, this study shows the up-regulation of the genes involved in ergosterol biosynthesis and sterol transport in C. glabrata cells in which sterol synthesis is defective or is abrogated by fluconazole treatment. We also corroborate that the sterol influx transporter CgAus1p imports exogenous cholesterol or ergosterol, which contributes to the development of clinical resistance to azole antifungals in C. glabrata. These findings demonstrate that sterol uptake and sterol biosynthesis may act coordinately and collaboratively to sustain growth and to mediate antifungal resistance in $C$. glabrata through dynamic gene expression in response to azole stress and environmental challenges.

\section{Acknowledgements}

The authors would like to thank Ms. Cindy Clark for carefully reviewing the manuscript. 


\section{Funding}

This study was supported by the Intramural Research Program of the National Institute of Allergy and Infectious Diseases, National Institutes of Health.

\section{Availability of data and materials}

All data generated or analyzed during this study are included in this published article.

\section{Authors' contributions}

QQL conceived of the project, conducted the studies, performed drug sensitivity assay, RT-qPCR and Southern blot analysis, cloning of CgAUS1, construction of Cgergl and Cgausl double mutant, as well as other experiments, carried out the analysis and interpretation of data, wrote the manuscript, and is the primary author of this paper. HFT conceived the project, performed microarray hybridization and data analysis, and critically reviewed the manuscript. AM performed bioinformatics and statistical analysis. BAW, JAN, and YF analyzed and interpreted data and edited the manuscript. JEB participated in the design and coordination of the study, critically reviewed the manuscript, and edited the final version of the paper. All authors read and approved the final manuscript.

\section{Ethics approval and consent to participate}

Not applicable.

\section{Consent for publication}

Not applicable.

\section{Competing interests}

The authors declare that they have no competing interests.

\section{References}

1. Pappas PG, Kauffman CA, Andes DR, Clancy CJ, Marr KA, Ostrosky-Zeichner L, Reboli AC, Schuster MG, Vazquez JA, Walsh TJ, et al: Executive summary: Clinical practice guideline for the management of candidiasis: 2016 update by the infectious diseases society of America. Clin Infect Dis 62: 409-417, 2016.

2. Rodrigues CF, Silva S and Henriques M: Candida glabrata: A review of its features and resistance. Eur J Clin Microbiol Infect Dis 33: 673-688, 2014.

3. Inukai T, Nagi M, Morita A, Tanabe K, Aoyama T, Miyazaki Y, Bard $M$ and Nakayama $H$ : The mannoprotein TIR3 (CAGL0C03872g) is required for sterol uptake in Candida glabrata. Biochim Biophys Acta 1851: 141-151, 2015.

4. Nagi M, Nakayama H, Tanabe K, Bard M, Aoyama T. Okano M, Higashi S, Ueno K, Chibana H, Niimi M, et al: Transcription factors CgUPC2A and CgUPC2B regulate ergosterol biosynthetic genes in Candida glabrata. Genes Cells 16: 80-89, 2011.

5. Nagi M, Tanabe K, Ueno K, Nakayama H, Aoyama T, Chibana H, Yamagoe S, Umeyama T, Oura T, Ohno $\mathrm{H}$, et al: The Candida glabrata sterol scavenging mechanism, mediated by the ATP-binding cassette transporter Aus1p, is regulated by iron limitation. Mol Microbiol 88: 371-381, 2013.
6. Silver PM, Oliver BG and White TC: Role of Candida albicans transcription factor Upc2p in drug resistance and sterol metabolism. Eukaryot Cell 3: 1391-1397, 2004.

7. MacPherson S, Akache B, Weber S, De Deken X, Raymond M and Turcotte B: Candida albicans zinc cluster protein Upc2p confers resistance to antifungal drugs and is an activator of ergosterol biosynthetic genes. Antimicrob Agents Chemother 49: $1745-1752,2005$

8. Tsai HF, Bard M, Izumikawa K, Krol AA, Sturm AM, Culbertson NT, Pierson CA and Bennett JE: Candida glabrata erg1 mutant with increased sensitivity to azoles and to low oxygen tension. Antimicrob Agents Chemother 48: 2483-2489, 2004.

9. Bard M, Sturm AM, Pierson CA, Brown S, Rogers KM, Nabinger S, Eckstein J, Barbuch R, Lees ND, Howell SA and Hazen KC: Sterol uptake in Candida glabrata: Rescue of sterol auxotrophic strains. Diagn Microbiol Infect Dis 52: 285-293, 2005.

10. Flowers SA, Colón B, Whaley SG, Schuler MA and Rogers PD: Contribution of clinically derived mutations in ERG11 to azole resistance in Candida albicans. Antimicrob Agents Chemother 59: 450-460, 2015.

11. Li QQ, Skinner J and Bennett JE: Evaluation of reference genes for real-time quantitative PCR studies in Candida glabrata following azole treatment. BMC Mol Biol 13: 22, 2012.

12. Vermitsky JP, Earhart KD, Smith WL, Homayouni R, Edlind TD and Rogers PD: Pdr1 regulates multidrug resistance in Candida glabrata: Gene disruption and genome-wide expression studies. Mol Microbiol 61: 704-722, 2006.

13. Shannon P, Markiel A, Ozier O, Baliga NS, Wang JT, Ramage D, Amin N, Schwikowski B and Ideker T: Cytoscape: A software environment for integrated models of biomolecular interaction networks. Genome Res 13: 2498-2504, 2003.

14. Bindea G, Mlecnik B, Hackl H, Charoentong P, Tosolini M, Kirilovsky A, Fridman WH, Pagès F, Trajanoski Z and Galon J: ClueGO: A Cytoscape plug-in to decipher functionally grouped gene ontology and pathway annotation networks. Bioinformatics 25: 1091-1093, 2009.

15. Bindea G, Galon J and Mlecnik B: CluePedia Cytoscape plugin: Pathway insights using integrated experimental and in silico data. Bioinformatics 29: 661-663, 2013.

16. van den Hazel HB, Pichler H, do Valle Matta MA, Leitner E, Goffeau A and Daum G: PDR16 and PDR17, two homologous genes of Saccharomyces cerevisiae, affect lipid biosynthesis and resistance to multiple drugs. J Biol Chem 274: 1934-1941, 1999.

17. Marek M, Milles S, Schreiber G, Daleke DL, Dittmar G, Herrmann A, Müller P and Pomorski TG: The yeast plasma membrane ATP binding cassette $(\mathrm{ABC})$ transporter Aus1: Purification, characterization, and the effect of lipids on its activity. J Biol Chem 286: 21835-21843, 2011.

18. Whaley SG, Caudle KE, Vermitsky JP, Chadwick SG, Toner G, Barker KS, Gygax SE and Rogers PD: UPC2A is required for high-level azole antifungal resistance in Candida glabrata. Antimicrob Agents Chemother 58: 4543-4554, 2014.

19. Ness F, Bourot S, Régnacq $M$, Spagnoli R, Bergès $T$ and Karst F: SUT1 is a putative Zn[II]2Cys6-transcription factor whose upregulation enhances both sterol uptake and synthesis in aerobically growing Saccharomyces cerevisiae cells. Eur J Biochem 268: 1585-1595, 2001.

20. Zavrel M, Hoot SJ and White TC: Comparison of sterol import under aerobic and anaerobic conditions in three fungal species, Candida albicans, Candida glabrata, and Saccharomyces cerevisiae. Eukaryot Cell 12: 725-738, 2013.

21. Nakayama H, Tanabe K, Bard M, Hodgson W, Wu S, Takemori D, Aoyama T, Kumaraswami NS, Metzler L, Takano Y, et al: The Candida glabrata putative sterol transporter gene CgAUS1 protects cells against azoles in the presence of serum. J Antimicrob Chemother 60: 1264-1272, 2007.

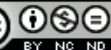

This work is licensed under a Creative Commons Attribution-NonCommercial-NoDerivatives 4.0 International (CC BY-NC-ND 4.0) License. 